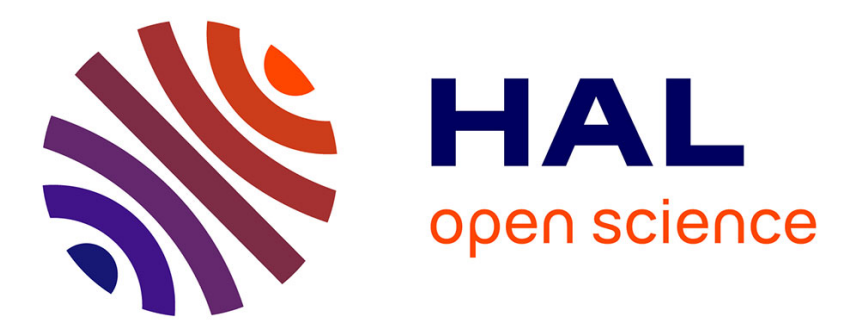

\title{
Statistical assessment of multiaxial HCF criteria at the grain scale
}

Anis Hor, Nicolas Saintier, Camille Robert, Thierry Palin-Luc, Franck Morel

\section{To cite this version:}

Anis Hor, Nicolas Saintier, Camille Robert, Thierry Palin-Luc, Franck Morel. Statistical assessment of multiaxial HCF criteria at the grain scale. International Journal of Fatigue, 2014, 67, pp.151-158. 10.1016/j.ijfatigue.2014.01.024 . hal-01057879

\section{HAL Id: hal-01057879 https://hal.science/hal-01057879}

Submitted on 23 Apr 2015

HAL is a multi-disciplinary open access archive for the deposit and dissemination of scientific research documents, whether they are published or not. The documents may come from teaching and research institutions in France or abroad, or from public or private research centers.
L'archive ouverte pluridisciplinaire HAL, est destinée au dépôt et à la diffusion de documents scientifiques de niveau recherche, publiés ou non, émanant des établissements d'enseignement et de recherche français ou étrangers, des laboratoires publics ou privés. 


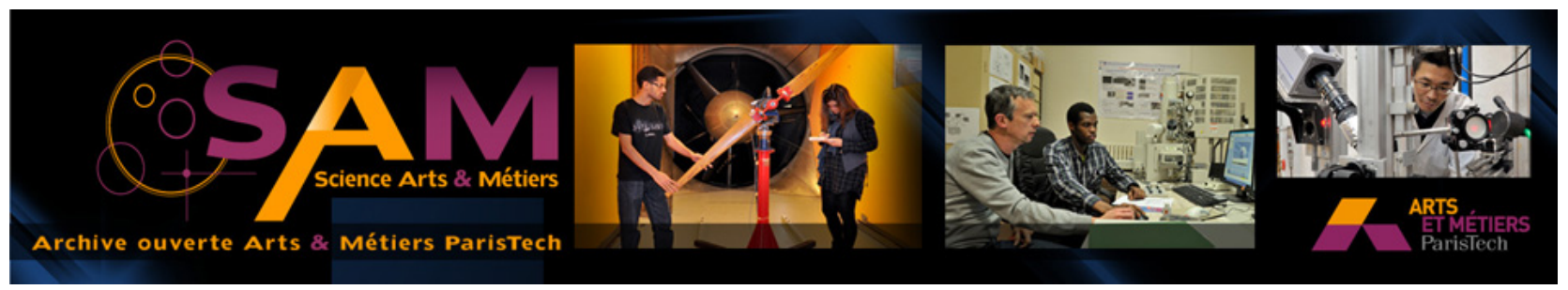

Science Arts \& Métiers (SAM)

is an open access repository that collects the work of Arts et Métiers ParisTech researchers and makes it freely available over the web where possible.

This is an author-deposited version published in: http://sam.ensam.eu

Handle ID: .http://hdl.handle.net/10985/8394

\section{To cite this version :}

Franck MOREL, Anis HOR, Thierry PALIN-LUC, Nicolas SAINTIER, Camille ROBERT - Statistical assessment of multiaxial HCF criteria at the grain scale - International Journal of Fatigue - Vol. 67, p.151-158 - 2014 


\title{
Statistical assessment of multiaxial HCF criteria at the grain scale
}

\author{
Anis Hor ${ }^{\mathrm{a}, *}$, Nicolas Saintier ${ }^{\mathrm{a}}$, Camille Robert $^{\mathrm{b}}$, Thiery Palin-Luc ${ }^{\mathrm{a}}$, Franck Morel ${ }^{\mathrm{b}}$ \\ ${ }^{a}$ Arts et Métiers ParisTech, I2M, CNRS, Esplanade des Arts et Métiers, 33405 Talence, France \\ ${ }^{\mathrm{b}}$ Arts et Métiers ParisTech, LAMPA - 2 Bd du Ronceray, 49035 Angers, France
}

Keywords:

Multiaxial HCF

Copper

Crystal plasticity

Extreme value probability

FEA

\begin{abstract}
A B S T R A C T S
Multiaxial high cycle fatigue modeling of materials is an issue that concerns many industrial domains (automotive, aerospace, nuclear, etc.) and in which many progress still remains to be achieved. Several approaches exist in the literature: invariants, energy, integral and critical plane approaches all of them having their advantages and drawbacks. These different formulations are usually based on mechanical quantities at the micro or mesoscales using localization schemes and strong assumptions to propose simple analytical forms. This study aims to revisit these formulations using a numerical approach based on crystal plasticity modeling coupled with explicit description of microstructure (morphology and texture) and proposes a statistical procedure for the analyses of numerical results in the HCF context. This work has three steps: First, 2.5D periodic digital microstructures based on a random grain sizes distribution are generated. Second, multiaxial cyclic loading conditions corresponding to the fatigue strength at 106 cycles are applied to these microstructures. Third, the mesoscopic Fatigue Indicator Parameters (FIPs), formulated from the different criteria existing in the literature, are identified using the finite element calculations of the mechanical fields. These mesoscopic FIP show the limits of the original criteria when it comes to applying them at the grain scale. A statistical method based on extreme value probability is used to redefine the thresholds of these criteria. These new thresholds contain the sensitivity of the HCF behavior to microstructure attributes. Finally, the biaxiality and phase shift effects are discussed at the grain scale and the loading paths of some critical grains are analyzed.
\end{abstract}

\section{Introduction}

It is generally accepted that the spread of the macroscopic fatigue strength in metals (determined by the initiation of a technical crack having a typical size about $1 \mathrm{~mm}$ ) is a consequence of the microstructural heterogeneities [1,2]. Indeed, in the HCF regime, the imposed stress amplitude is most of the time smaller than the macroscopic cyclic yield stress of the material, therefore plasticity is highly heterogeneous and localized. The fatigue strength can vary from one specimen to another due to the microstructure variability (texture, grain morphology, phase, defect, etc.). To take into account the role of microstructural heterogeneities in multiaxial fatigue prediction, recent models have been developed in a probabilistic framework [3,4]. These models

\footnotetext{
Abbreviations: HCF, high cycle fatigue; FIP, Fatigue Indicator Parameter; RVE, representative volume element; SVE, Statistical Volume Element; GEV, generalized extreme value distribution; Microscopic scale, Corresponding to the integration points; Mesoscopic scale, corresponding to the average density in a grain; Macroscopic scale, Corresponding to the elementary volume average.

* Corresponding author. Tel.: +33 (0)556845371; fax: +33 (0)556845366.

E-mail address: anis.hor@ensam.eu (A. Hor).
}

allowed to establish a relation between the microstructure components and the variability of the fatigue behavior using generally the weakest link hypothesis [5,6]. Although, these proposed criteria give interesting results, they do not allow to consider simultaneously the different components of the microstructure as well as their interactions (e.g. the texture effect). In order to consider these effects in the case of HCF behavior, recent studies have focused on numerical simulations based on explicit modeling of microstructure coupled with the multi-scale modeling of the fatigue behavior based on the crystal plasticity to connect the variability of the fatigue strength to the microstructure heterogeneity $[1,7,8]$. This variability can be described by the extreme value probability of the representative parameters of the microcrack initiation. Recently, Przybyla et al. [2,9] introduced a new framework to analyze the microstructure sensitivity of the fatigue strength. This method takes into account neighborhood effects with the extreme values of the marked correlation functions [10]. This enabled the authors to quantify the interactions between the microstructure attributes and the fatigue strength. The authors used the Gumbel extreme value distribution to describe the microstructure sensitivity of the 
Fatemi and Socie fatigue indicator parameter [11] under uniaxial loading.

The proposed work is devoted to multiaxial HCF strength criteria. The purpose is, first, to analyze the microstructure sensitivity (grain morphology and orientation) of the FIPs corresponding to the adaptation of several multiaxial HCF strength criteria at the mesoscopic scale. A statistical study is done to define new mesoscopic thresholds for these FIPs. Finally, the capability of the criteria, applied at the macroscopic scale, to take into account the microstructure sensitivity is discussed through a comparison between the thresholds determined at grain scale (called mesoscopic) by the generalized extreme value (GEV) distribution and the original macroscopic thresholds. This comparison concerns also the biaxiality and phase shift effect.

\section{Numerical model}

Finite element computations were used to compute strain and stress fields at the grain scale. Constitutive equations, grain morphology and orientation composing this model are described below.

\subsection{Constitutive relations}

The material studied in this work is pure copper. Its facecentered cubic (fcc) structure reduces the computation time due to the limited slip systems number (12 slip systems) compared to other microstructures. The elastic part of the behavior is considered as anisotropic (cubic elasticity) while the plastic part is modeled by crystal plasticity. The stiffness constants are $C_{11}=159 \mathrm{GPa}, C_{12}=$ $121.9 \mathrm{GPa}$ and $C_{44}=80.9 \mathrm{GPa}$ using Voigt notation. The crystal behavior used in this work was introduced by Meric and Cailletaud [12]. The constitutive relations are defined by Eqs. ((1)-(3)), where $\gamma^{s}$ is the plastic shear on the slip system $s, v^{s}$ is the cumulative plastic shear on the system $s, \tau^{s}$ is the resolved shear stress of the slip system $s$ and $\underline{\dot{\varepsilon}}^{p l}$ is the plastic strain rate tensor.

$\dot{\gamma}^{s}=\left\langle\frac{\left\|\tau^{s}-x^{s}\right\|-r^{s}}{K}\right\rangle^{n} \operatorname{sign}\left(\tau^{s}\right)=\dot{v}^{s} \operatorname{sign}\left(\tau^{s}\right)$,

$\tau^{s}=\underline{\underline{m}}^{s}: \underline{\underline{\sigma}}$,

$\underline{\underline{\dot{\varepsilon}}}^{p l}=\sum_{s} \dot{\gamma}^{s} \underline{\underline{m}}^{s}$, where $\langle a\rangle$ is the positive part of $a$. The orientation tensor $\underline{m}^{s}$ is calculated on each slip system $s$ by the tensorial product of the unit normal vector $\underline{n}^{s}$ orientating the slip plane and the unit vector $\underline{l}^{s}$ orientating the slip direction:

$\underline{\underline{m}}^{s}=\frac{1}{2}\left(\underline{n}^{s} \otimes \underline{l}^{s}+\underline{l}^{s} \otimes \underline{n}^{s}\right)$

The twelve $<111>\{110\}$ slip systems of fcc structure are considered. The evolution of the isotropic hardening variable $r^{s}$ takes into account slip system interactions by means of the interaction matrix [h] defined by Franciosi et al. [13], with components $h_{r}^{s}$ :

$r^{s}=r_{0}+Q \sum_{r} h_{r}^{s}\left(1-\exp \left(-b v^{s}\right)\right)$

The kinematic hardening is described on each slip system by:

$x^{s}=c \alpha^{s} \quad$ with $\dot{\alpha}^{s}=\dot{\gamma}^{s}-d \dot{v}^{s} \alpha^{s}$.

Material parameters $K, n, r_{0}, Q, b, c, d$ and the interaction matrix components $\left(h_{r}\right)$ have been identified from experimental data on a high purity copper presented in $[14,15]$, the values are summarized in Table 1.

\subsection{Grain morphology and crystallographic texture}

The FE simulations performed in this work were done using 2.5D periodic microstructures [16]. The method used to create the topology of the aggregates was based on random distributions in size and shape of ellipses. The geometrical model was discretized by 32,000 linear triangular finite elements with the generalized plane strain assumption. Computed microstructure contains 200 equiaxed grains (see Fig. 1), with an average of 160 finite elements per grain. Such grain paving was found to be a good compromise between adequate description of the local strain/stress field and reasonable computation time. Finally, the selection of 200 crystal orientations was carried out in the Euler space defined by the three angles $\left(\varphi_{1}, \phi, \varphi_{2}\right)$ assuming cubic crystal symmetry and triclinic sample symmetry. The set of crystal orientations was performed so that, given the low number of orientations, this crystallographic aggregate can be considered as having no preferential orientations (isotropic texture).

Table 1

Material parameters for pure copper (from [14,15]).

\begin{tabular}{|c|c|c|c|c|c|c|c|c|c|c|c|c|}
\hline$K\left(\operatorname{MPa~s}{ }^{1 / n}\right)$ & $n$ & $r_{0}(\mathrm{MPa})$ & $Q(\mathrm{MPa})$ & $b$ & $c(\mathrm{MPa})$ & $d$ & $h_{0}$ & $h_{1}$ & $h_{2}$ & $h_{3}$ & $h_{4}$ & $h_{5}$ \\
\hline 8 & 20 & 15 & 4 & 12 & 32,000 & 900 & 1 & 1 & 0.2 & 90 & 3 & 2.5 \\
\hline
\end{tabular}

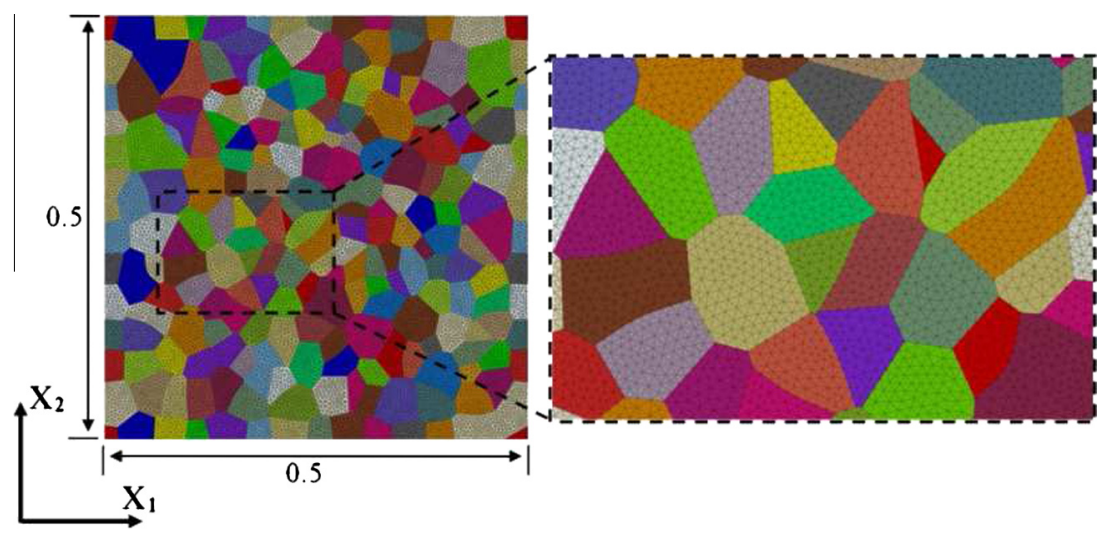

Fig. 1. Grain morphology and mesh. 


\subsection{Fatigue loading conditions}

Different loading conditions are investigated in this study: uniaxial loadings, and tension/torsion fully reversed loadings with different biaxiality ratios $k=\frac{T_{a}}{\Sigma_{a}}$ and different phase shifts. The combined loading levels equivalent to the fatigue limit at $10^{6}$ cycles are determined using Crossland criterion [17]. These load levels are given in Table 2.

\section{Mesoscopic Fatigue Indicator Parameters}

In this paper, two different formulations of stress-based criteria were considered and compared:

- A global formulation using an averaged stress: the octahedral shear stress in the Crossland [17] criterion.

- A critical plane formulation, this is the case of Matake [18] and Dang Van $[19,20]$ criteria. The latter contains a more physical formulation based on a mesoscopic approach with analytical scale transition (Lin-Taylor hypothesis).

Similar approach on energy or strain/stress based criteria are under investigation and will be presented in a fore coming paper.

These fatigue criteria are generally defined in the context of continuum mechanics. In order to evaluate the fatigue criterion on each computed microstructure, the usual HCF criteria are projected on each slip system of the crystals (Fig. 2). This procedure

Table 2

Biaxiality ratio $k$, tension stress amplitude, $\Sigma_{a}$ (MPa), shear stress amplitude, $T_{a}$ (MPa) and phase shift $\varphi\left(^{\circ}\right)$ used for the different fully reversed loading conditions.

\begin{tabular}{|c|c|c|c|c|c|c|c|c|c|}
\hline \multicolumn{10}{|c|}{ Uniaxial loadings } \\
\hline \multicolumn{4}{|l|}{$k$} & \multicolumn{4}{|c|}{$\Sigma_{a}(\mathrm{MPa})$} & \multicolumn{2}{|c|}{$T_{a}(\mathrm{MPa})$} \\
\hline 0 & & & & 56 & & & & 0 & \\
\hline$\infty$ & & & & 0 & & & & 36 & \\
\hline \multicolumn{10}{|c|}{ Multiaxial loadings } \\
\hline$k$ & $\begin{array}{l}\sum_{a} \\
(\mathrm{MPa})\end{array}$ & $\begin{array}{l}T_{a} \\
(\mathrm{MPa})\end{array}$ & $\begin{array}{l}\varphi \\
\left(^{\circ}\right)\end{array}$ & $\begin{array}{l}\sum_{a} \\
(\mathrm{MPa})\end{array}$ & $\begin{array}{l}T_{a} \\
(\mathrm{MPa})\end{array}$ & $\begin{array}{l}\varphi \\
\left(^{\circ}\right)\end{array}$ & $\begin{array}{l}\sum_{a} \\
(\mathrm{MPa})\end{array}$ & $\begin{array}{l}T_{a} \\
(\mathrm{MPa})\end{array}$ & $\begin{array}{l}\varphi \\
\left(^{\circ}\right)\end{array}$ \\
\hline 0.25 & 52 & 13 & 0 & 54 & 13.5 & 45 & 56 & 14 & 90 \\
\hline 0.5 & 43.5 & 22 & 0 & 47 & 23.5 & 45 & 56 & 28 & 90 \\
\hline 0.75 & 36 & 27 & 0 & 38.5 & 29 & 45 & 44.5 & 33 & 90 \\
\hline 1 & 29.5 & 29.5 & 0 & 31 & 31 & 45 & 34 & 34 & 90 \\
\hline 2 & 17 & 34 & 0 & 17 & 34.5 & 45 & 17.5 & 35 & 90 \\
\hline
\end{tabular}

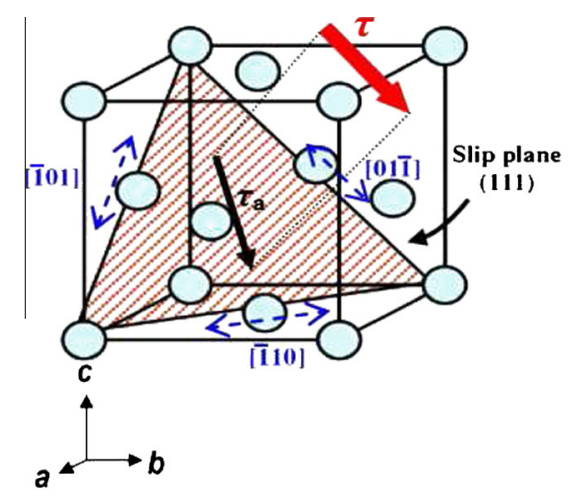

is repeated for each crystal considering its local orientation $\left(\varphi_{1}, \phi, \varphi_{2}\right)$ and stress state computed by FEA for each loading case. For instance, the shear stress vector in a given plane (mechanical quantity introduced in the computation of the above cited criteria) is transformed into a resolved shear stress vector over a slip system. The rotation of the crystal in the physical space (defined by the Euler angles $\left.\left(\varphi_{1}, \phi, \varphi_{2}\right)\right)$ covers all the planes and directions of space, which enables to find the same critical planes and directions (planes and directions maximizing criterion) as those obtained by the original criterion (with continuous formulation). Table 3 gives the expressions of FIPs adapted at the crystal scale.

Finally, the parameters $\alpha_{i}$ and $\beta_{i}$ describing the median macroscopic threshold of the considered criteria are identified from two median fatigue limits at $10^{6}$ cycles obtained on smooth specimens under fully reversed loadings: tension $\left(s_{-1}=56.2 \mathrm{MPa}\right)$ and torsion $\left(t_{-1}=36.1 \mathrm{MPa}\right)$ taken from the work of Lukas and Kunz [21] on pure copper. The expression of $\alpha_{i}$ and $\beta_{i}$ are also given in Table 3.

\section{Microstructure sensitivity}

Fig. 3 compares the mesoscopic Dang Van FIP predictions and the macroscopic criterion (solid line). This comparison shows the existence of grains for which the FIP exceeded the macroscopic threshold. This results show that the macroscopic threshold is not applicable at the grain scale despite the fact that this criterion is based on micro-macro scale transition and such is considered as evaluating the mechanical quantities at this scale. It evidences that numerical simulations at the grain scale provide a more efficient way to access grain scale quantities by describing neighboring effects and local anisotropy. As shown by Robert et al. [16], both shear and hydrostatic terms deviate from the values predicted by the homogenization scale transition due to elastic anisotropy and, in a less pronounced way, crystal plasticity. A new mesoscopic threshold (called $\beta^{\prime}$ ) can be defined, which corresponds to the line linking the most loaded (critical) grains (plotted in red in the graphs of Fig. 3). However, this determination method of the new mesoscopic threshold is ambiguous since it considers that the studied elementary volume is representative with regards to fatigue. This hypothesis is not acceptable in the case of a non-deterministic behavior such as HCF strength. This is verified by adding the FIP values (plotted in blue circle in Fig. 3) obtained by the same procedure applied to a second VE. New critical grains are obtained with FIPs values above the threshold defined from the first FEA applied to the first VE (see Fig. 3). The RVE hypothesis has to be replaced by a statistical analysis of the extreme FIP value response

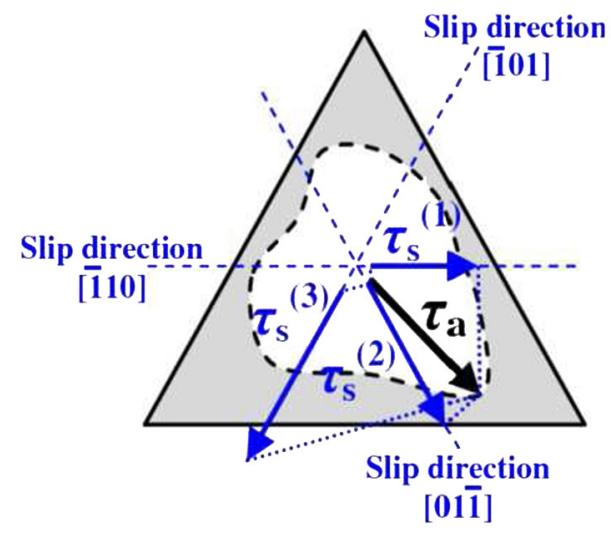

Fig. 2. Schematization of the shear stress vector projection on the slip systems of the FCC crystal. 
Table 3

Expression of the Fatigue Indicator Parameters (FIPs) of the studied criteria.

\begin{tabular}{|c|c|c|c|}
\hline Criterion & $I_{i}$ & $\alpha_{i}$ & $\beta_{i}$ \\
\hline Crossland & $I_{c r}=\tau_{o c t, a}^{s}+\alpha_{c r} \sigma_{h y d, \max } \leqslant \beta_{c r}$ & $\alpha_{c r}=\frac{t_{-1}-S_{-1} / \sqrt{3}}{S_{-1} / 3}$ & $\beta_{c r}=t_{-1}$ \\
\hline Matake & $I_{m}=\max _{s=1,12}\left(\tau_{a}^{s}\right)+\alpha_{m} \sigma_{s *, \max } \leqslant \beta_{m}$ & $\alpha_{m}=2 \frac{t_{-1}}{s_{-1}}-1$ & $\beta_{m}=t_{-1}$ \\
\hline Dang Van & $I_{d v}=\max _{s=1,12}\left\{\max _{t}\left[\left\|\underline{\hat{\tau}}^{s}(s, t)\right\|+\alpha_{d v} \hat{\sigma}_{h y d}(t)\right]\right\} \leqslant \beta_{d v}$ & $\alpha_{d v}=\frac{t_{-1}-s_{-1} / 2}{s_{-1} / 3}$ & $\beta_{d v}=t_{-1}$ \\
\hline
\end{tabular}

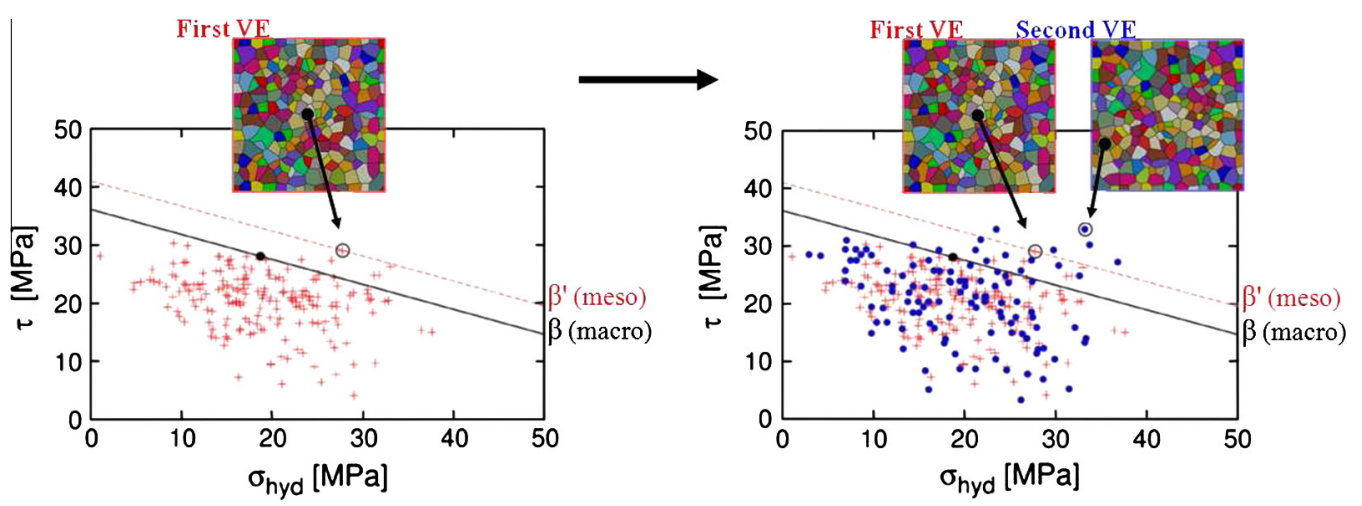

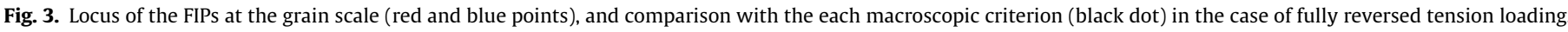

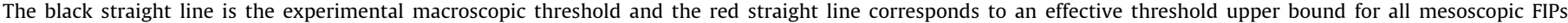
determined from a first VE. (For interpretation of the references to color in this figure legend, the reader is referred to the web version of this article.)

of the microstructure (described by a given set of attributes) for each studied FIPs.

In other words, the HCF strength is related to the critical grain whose response leads to the maximum value of the FIP. These extreme values are located at the tails of the density functions of mesoscopic responses and are highly sensitive to the microstructure attributes. To study these critical grains, several statistical approaches are possible. The method selected for this work is based on the extreme value probability following the pioneer work of Freudenthal and Gumbel [22] at the macroscale and Przybyla et al. [2] at the local scale.

The extreme value database was constructed by identifying the maximum value of FIP for each Statistical Volume Element (SVE) considering that the SVE is an RVE from the cyclic behavior point of view. For each loading case, 64 SVEs were considered: they were obtained by the combination of 8 random morphologies (equiaxed grains) and 8 isotropic textures. Such a size of SVE set was found to be sufficient to stabilize the extreme value distribution parameters identification.

\section{Generalized extreme values probability}

As a common practice in fatigue, the Gumbel distribution is commonly used $[1,9,22]$ for describing extreme values distributions without justification. The challenge in applying extreme value statistics is to determine the type of distribution that is representative of the considered statistical variable. In this work a generalized extreme value distribution is used to avoid choosing a priori the distribution type.

Let us consider a random variable $X$ with a cumulative distribution function $F_{X}(x)=P(X \leqslant x)$. The $n$ extreme realizations, $Y_{n}$, in $n$ samples of the random variable, $X$, can be defined as follows:

$Y_{n}=\max \left(X_{1}, X_{2}, \ldots, X_{n}\right)$.

The distribution function of $Y_{n}$ is defined in $x$ as follows:

$F_{Y_{n}}(x) \equiv P\left(Y_{n} \leqslant x\right)=P\left(X_{1} \leqslant x, X_{2} \leqslant x, \ldots, X_{n} \leqslant x\right)$.
According to the Fisher-Tippet theorem [23], if there exist two real normalizing sequences $\left(a_{n}\right)_{n \geqslant 1}>0$ and $\left(b_{n}\right)_{n \geqslant 1}$ and a nondegenerated distribution (i.e. not reduced to a single point) $G$ so that:

$P\left(\frac{Y_{n}-b_{n}}{a_{n}} \leqslant x\right)=\left[F\left(a_{n} x+b_{n}\right)\right]^{n} \longrightarrow_{n \mapsto+\infty} G(x)$

then the distribution function $G$ is necessarily one of the three types of distributions: Fréchet, Weibull or Gumbel.

Jenkinson [24] proposed a single parametric expression (Eq. (10)) which includes these three types of distribution functions. It depends on a single parameter $\xi$. This function is called Generalized Extreme Value (GEV) distribution:

$G E V: \quad G_{\xi}(x)= \begin{cases}\exp \left(-(1+\xi x)^{-\frac{1}{\xi}}\right) & \text { if } \xi \neq 0, \forall x / 1+\xi x>0, \\ \exp (-\exp (-x)) & \text { if } \xi=0 .\end{cases}$

The parameter $\xi$ is called the extreme index (or shape factor) of the GEV distribution. Its sign indicates the type of asymptotic extreme function: the function is a Gumbel distribution if $\xi=0$, a Fréchet one if $\xi>0$, or a Weibull one if $\xi<0$. The variable $x=\frac{Y_{n}-b_{n}}{a_{n}}$ is called the normalized maximum of the random variable $X$. $a_{n}$ and $b_{n}$ parameters are called scale factors of the distribution.

The extreme value distribution function of the FIPs listed in Table 3 are considered in this study. The scale factors $\left(a_{n}\right.$ and $\left.b_{n}\right)$ and the extreme index $\xi$ are determined using the maximum likelihood method with a confidence interval of $99 \%$. Fig. 4 shows a comparison between the determined distributions and the numerical data extracted from FE computations for the Crossland, Matake and Dang Van FIPs. The identified GEV density function and distribution function showed a good correlation with the probability density and the cumulative probability determined from the extreme values of FIPs database.

The mean value and 99\% confidence intervals of the extreme index evolution as a function of the loading case and FIP is illustrated in Fig. 5. In all cases the mean value of $\xi$ deviates significantly from zero value. However, when considering the confidence intervals, it 
(a) Crossland FIP: $I_{c r}$
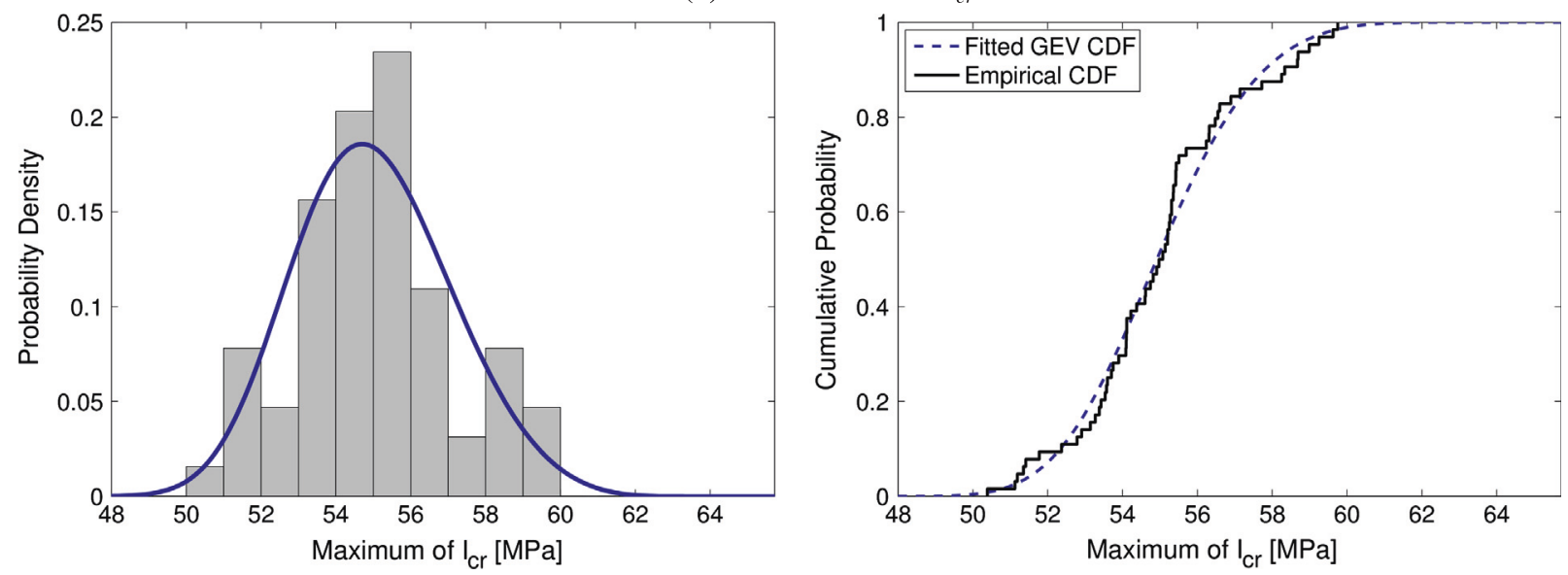

(b) Matake FIP: $I_{m}$
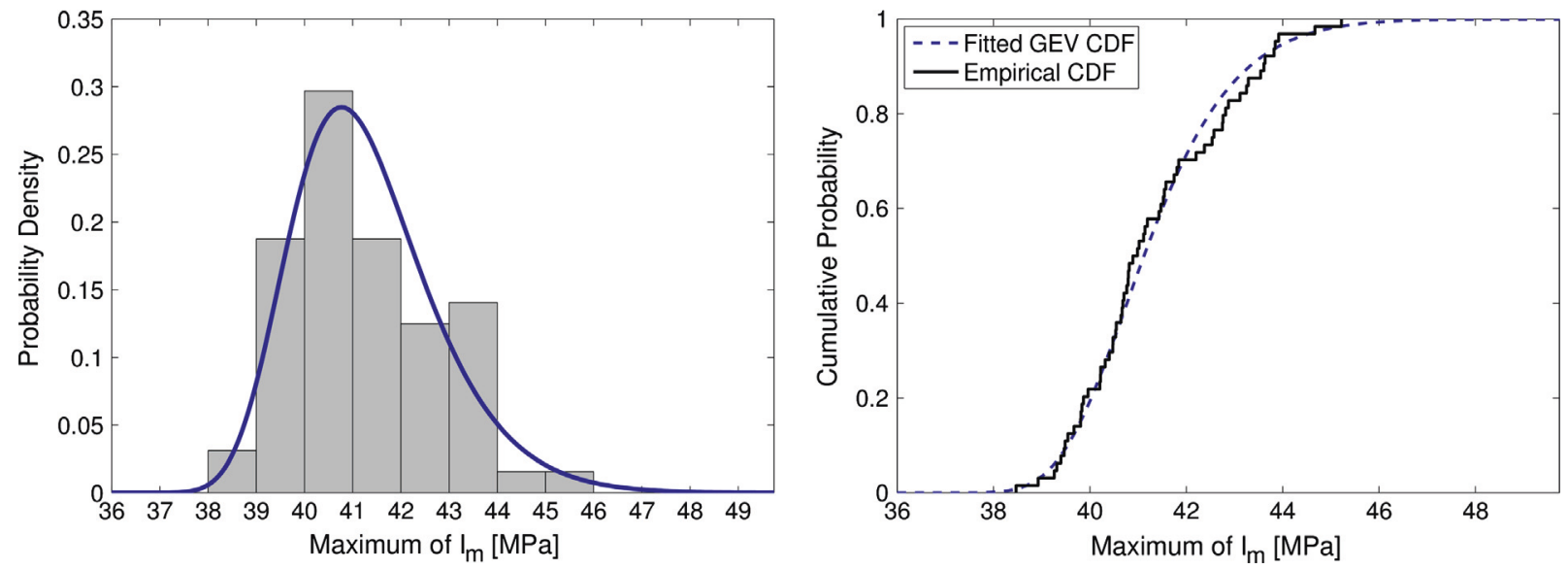

(c) Dang Van FIP: $I_{d v}$
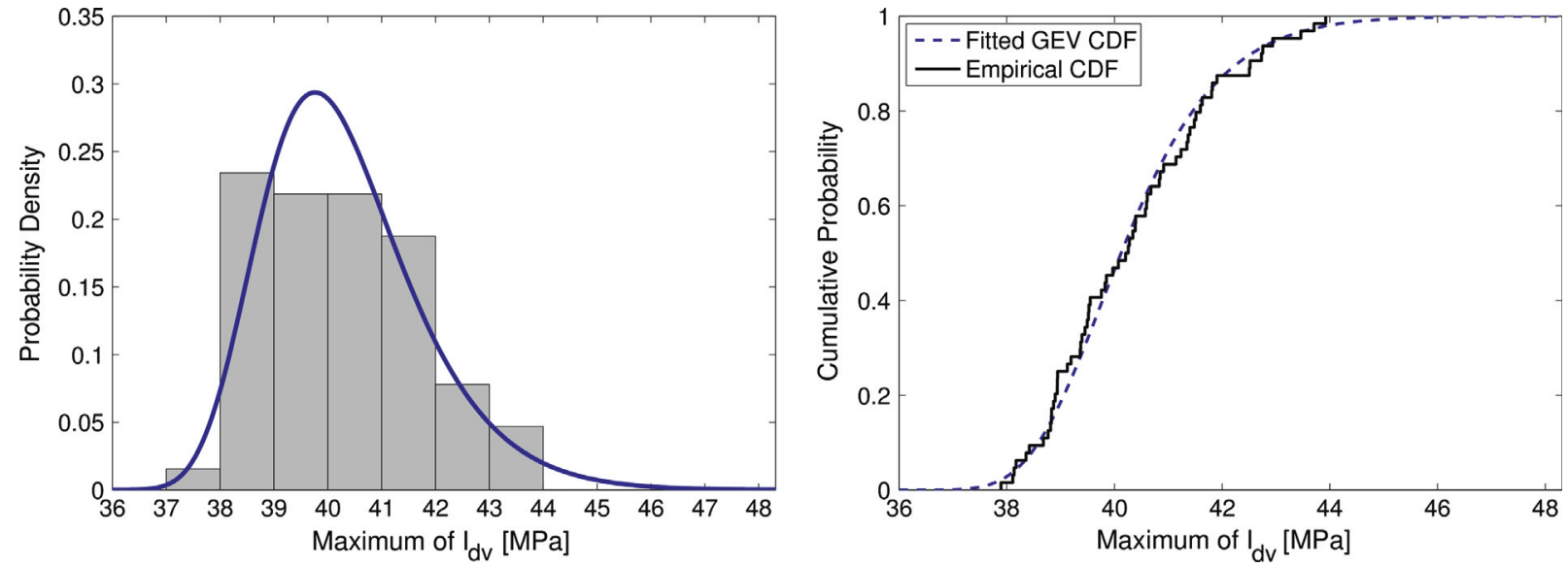

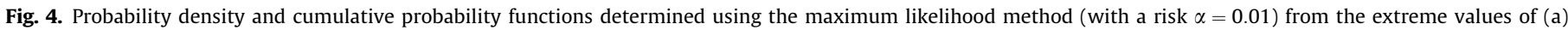
Crossland FIP, (b) Matake FIP et (c) Dang Van FIP for fully reversed tension loading with an amplitude $\left(\Sigma_{11}\right)_{a}=56.2 \mathrm{MPa}$.

seems reasonable to consider that a Gumbel distribution can efficiently describe the computed FIP extreme value distribution for most loading cases but not all.

One can note that $\xi=0$ (i.e. Gumbel distribution) is an acceptable value of the shape parameter for all the studied loading case in case of Matake and Dang Van FIPs. For some cases, the range of $\xi$ is unusually negative (e.g. Crossland FIP with TT0 $(k=0.25)$ and TT45 $(k=0.25)$ loadings) or positive (e.g. Crossland FIP with TT90 $(k=2)$ loading). Therefore the choice of the Gumbel distribution type can introduce significant error for these cases. Furthermore, there are no values of $\xi$ shared by all loading cases and all FIPs (Fig. 5).

\section{Results and discussions}

Fig. 6 represents the mesoscopic thresholds (medians and also the probabilities of 0.1 and 0.9 quintiles) for each loading 
(a) Crossland FIP

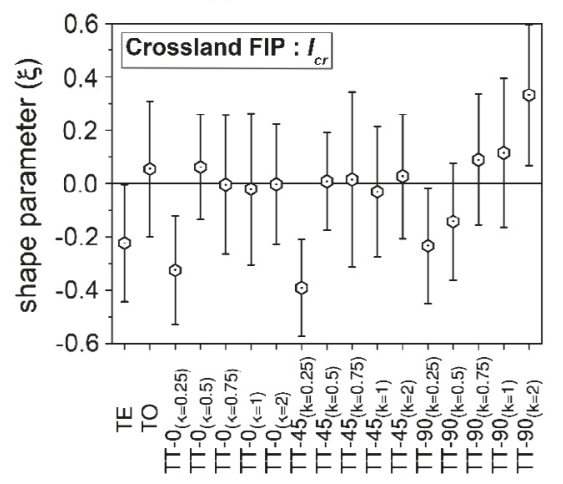

(b) Matake FIP

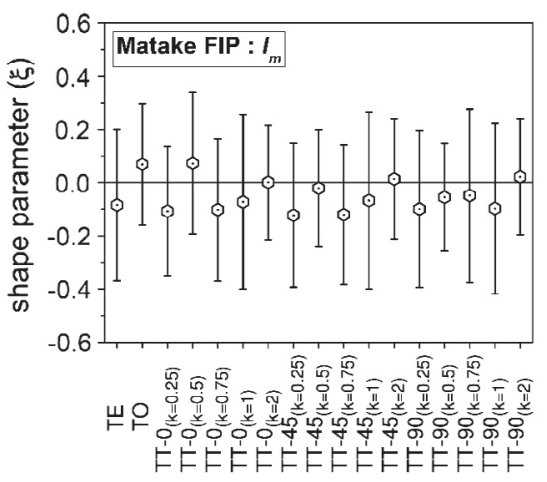

(c) Dang Van FIP

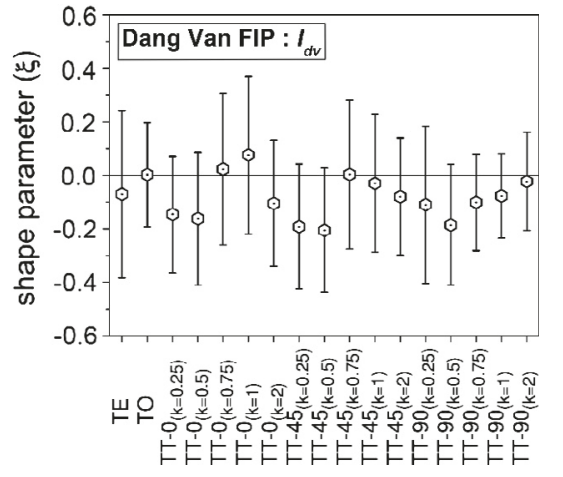

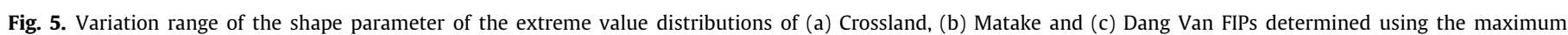
likelihood method (corresponding to a risk $\alpha=0.01$ ) for the studied loading conditions.

condition. These factors are normalized by the macroscopic threshold to analyze the effect of microstructure attributes and stress/ strain field heterogeneity at the grain scale. Referring to Fig. 5, for all studied loading conditions and studied FIPs, the normalized mesoscopic thresholds are always greater than 1. Applying of the studied criteria at the mesoscopic scale requires offsetting this threshold to account for microstructure effects at this length scale. It can be noticed that the local scale Crossland threshold is the one that deviates the most from the macroscopic one (ratio 1.5) compared to Dang Van (1.1) and Matake (1.17) threshold. This is due to the fact that the Crossland approach is based on an averaging procedure while Dang Van and Matake FIP formulations include a maximization procedure that makes the macroscale estimate closer to the extreme value analyses results. These results show that, in the case of isotropic texture, usual scale transition techniques can provide a reasonable estimate of the lower bound value of the extreme FIP values at the grain scale.

Fig. 6 illustrates also that the mesoscopic thresholds, defined as the medians of the extreme value distribution of the studied FIPs are sensitive to the loading case and such evidence a different sensitivity to multiaxiality compared to macroscopic threshold. This gap depends on the studied FIP: it is low in the case of the Crossland and Dang Van FIPs (Fig. 5a and c) but significant in the case of the Matake FIP (Fig. 5b). For this last FIP, the change in mesoscopic thresholds was observed especially for the biaxial loading with a phase shift of $90^{\circ}$.

The mesoscopic threshold, common to all loading cases was determined as the average of the thresholds associated to each loading conditions. This mesoscopic average threshold is shown in Fig. 6 by the dashed horizontal lines passing through all intervals bounded by the probabilities of 0.1 and 0.9 quintiles in the case of Crossland and Van Dang FIPs. For Matake FIP, for sake of simplicity such approach was also applied to the case of biaxial loadings with a phase shift of $90^{\circ}$ for Matake FIP. Further experimental work is needed to investigate such effects on this particular material.

Finally, assuming that the critical grains undergo the same loading path as the imposed loading on the entire aggregate, we determined the amplitudes of the stress $\Sigma_{11}$ and $\Sigma_{12}$ for the mesoscopic thresholds obtained by the extreme value statistics. These mesoscopic values are compared to the macroscopic one. Fig. 7 shows that the predictions of the biaxiality effects in mesoscopic and macroscopic scales are very similar for the three considered criteria. These trends are also found in the experimental studies in the literature $[25,26]$.

Fig. 8 illustrates the evolution of the amplitude of the normalized stress us a function of the phase shift. The three criteria overestimate the fatigue strength in the case of a non-zero phase shift (a) Crossland

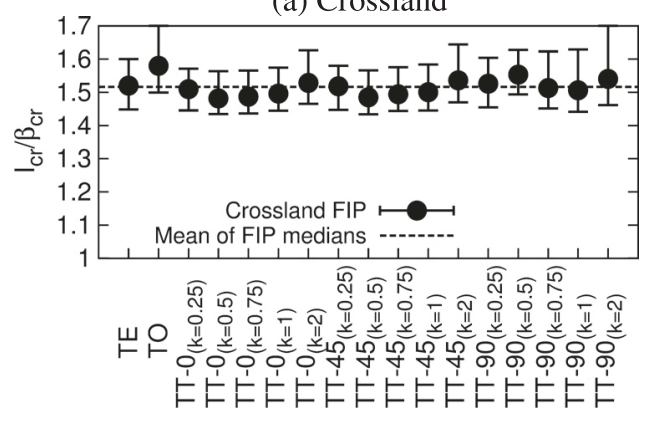

(b) Matake

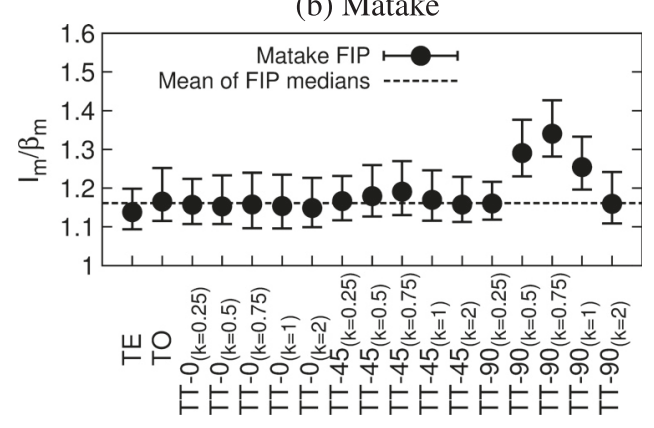

(c) Dang Van

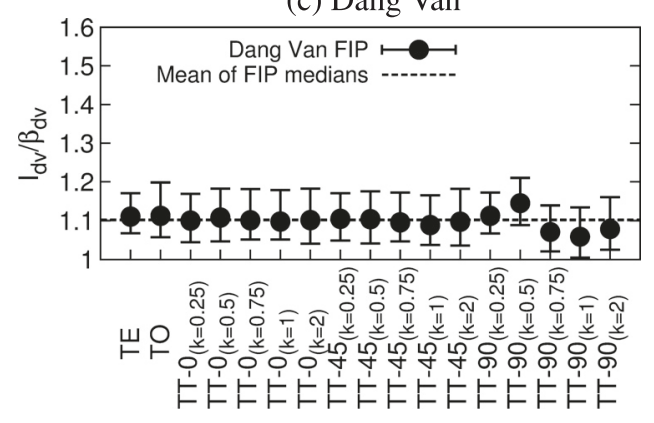

Fig. 6. Evolution of the median of the extreme value distributions of (a) Crossland (b) Matake and (c) Dang Van FIPs as a function of loading conditions. The black dots correspond to the median (probability of 0.5 ), the two limits of the interval correspond to a probability of 0.1 and 0.9 (i.e. $80 \%$ of the results are within this interval).

to both macroscopic and mesoscopic scales. These trends are also encountered in the literature especially in the case of the Dang Van criterion [3]. 
(a) Crossland FIP

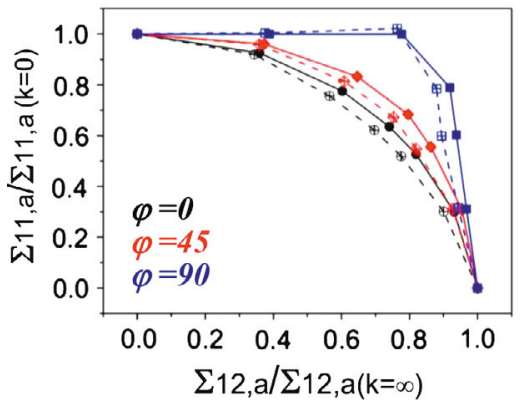

(b) Matake FIP

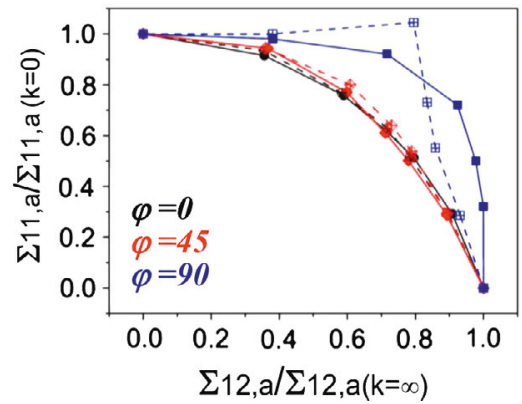

(c) Dang Van FIP

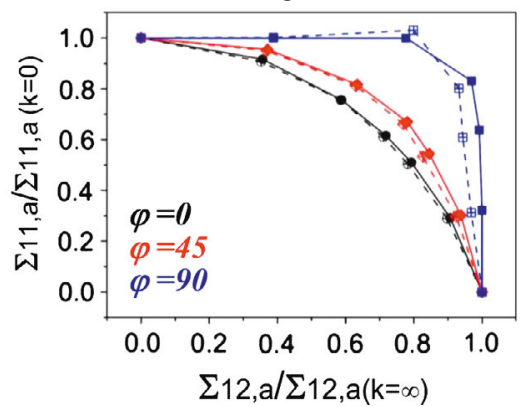

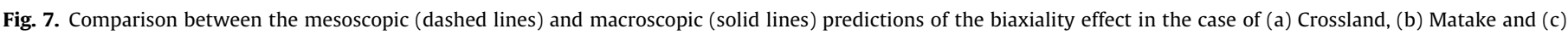
Dang Van criterion.

(a) Crossland FIP

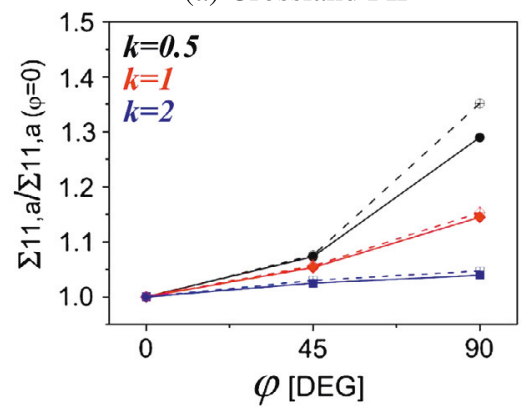

(b) Matake FIP

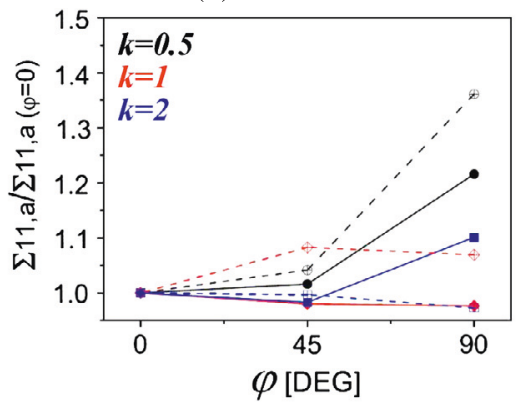

(c) Dang Van FIP

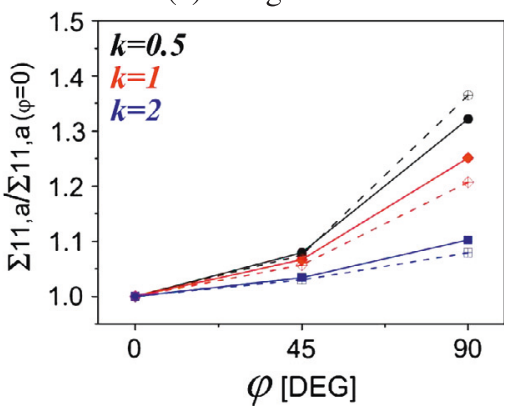

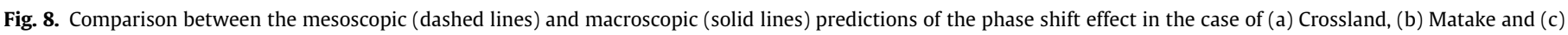
Dang Van criterion.

\section{Conclusion and prospects}

In this work, the responses of high cycle multiaxial fatigue criteria, widely studied in the literature, have been analyzed at the grain scale from polycrystalline modeling of pure copper recrystallized microstructure coupled with a statistical study of the critical grains. This statistical study allows the authors to introduce the microstructural heterogeneities effect in the variability of fatigue limits for proportional and non-proportional loadings. Some stress based multiaxial HCF strength criteria commonly used in the literature were adapted (i.e. modified) at the mesoscopic scale: Crossland, Matake and Dang Van. The comparison between the mesoscopic predictions of these criteria (named Fatigue Indicator Parameters, FIPs) and the macroscopic (original) criteria shows that they are not conservative at the grain scale. Indeed the identification of the macroscopic parameters of these criteria $\left(\alpha_{i}\right.$ and $\left.\beta_{i}\right)$ does not take into account the microstructure effects. The solution would be to readjust these parameters on the most critical grain predictions from a calculation on a representative volume element (RVE) according to the fatigue behavior.

However, on the one hand, the determination of the RVE is very difficult (probably impossible) in the case of a non-deterministic phenomenon so that fatigue where the behavior is very sensitive to microstructural heterogeneities. In this work, the RVE has been substituted by a statistical study on the so-called Statistical Volume Elements (SVE) [9].

Moreover, in fatigue researchers it has been focus generally on the critical grains (with a maximum FIP). These critical grains are situated in the tails of the aggregate response distributions. One of the most used methods to statistically study these critical grains is the extreme value statistics. A database of the extreme values of FIPs was therefore built. This database was resulting from FE calculations on 64 SVEs formed by 200 grains. For each SVE, several loading conditions (17 loading cases) were applied during ten cycles. As a result of this study it has been shown that the extreme value density probabilities of the various FIPs can be adequately represented by the Generalized Extreme Value (GEV) distribution.

The statistical moments determination of the different distributions allowed the definition of new thresholds called "mesoscopic" ones for the studied criteria. These thresholds are the average of the medians of the extreme value distributions related to the different loading conditions. According to the criteria, these thresholds are different or similar to the macroscopic thresholds. For the Dang Van criterion, the mesoscopic threshold is close to the macroscopic one. At the opposite, for the Crossland criterion, the ratio between meso and macro thresholds is greater than 1.5. Whereas for the Matake FIP the threshold ratio is around 1.2.

Except for the non-proportional biaxial loading with a phase shift of $90^{\circ}$, where applied loading levels are very different from one criterion to another, the mesoscopic thresholds is almost the same for all the loading conditions. These new mesoscopic thresholds can therefore be determined by FEA on a polycrystal aggregate under a single loading case. The obtained results are different when considering texture effects and will be presented in a forecoming paper.

This study has to be prolongated by studying other polycrystal aggregate (bcc, hcc for instance) to confirm (or not) these results. This work can also be extended to develop physical based HCF criteria, that consider a real modeling of the slip plane activation and the interaction between activated planes. Analyzing the effect of material microstructural defects (pores, inclusions) would be also a very interesting next step for providing robust criteria of multiaxial fatigue strength which are explicitly defects sensitive. Finally, the great increase of computational power allow the authors to imagine in future the possibility to simulate complex microstructure (multiphase, including defects so that microshrinkage, 
non-metallic inclusions). This should help scientists to better describe the complexity of the metal fatigue strength with (simple) local criterion.

\section{Acknowledgement}

Financial support of this research by Arts et Métiers Paristech is gratefully acknowledged.

\section{References}

[1] McDowell DL, Dunne FPE. Microstructure-sensitive computational modeling of fatigue crack formation. Int J Fatigue 2010;32(9):1521-42.

[2] Przybyla CP, Prasannavenkatesan R, Salajegheh N, McDowell DL. Microstructure-sensitive modeling of high cycle fatigue. Int J Fatigue 2010; 32(3):512-25.

[3] Morel F, Huyen N. Plasticity and damage heterogeneity in fatigue. Theor Appl Fract Mech 2008;49(1):98-127.

[4] Poncelet M, Doudard C, Calloch S, Weber B, Hild F. Probabilistic multiscale models and measurements of self-heating under multiaxial high cycle fatigue. J Mech Phys Solids 2010;58(4):578-93.

[5] Weibull W. A statistical theory of the strength of materials. The royal Swedish institute for engineering research, report; 1939.

[6] Weibull W. A statistical distribution function of wide applicability. ASME - J Appl Mech 1951;18:293-7.

[7] Bennett VP, McDowell DL. Polycrystal orientation distribution effects on microslip in high cycle fatigue. Int J Fatigue 2003;25(1):27-39.

[8] Guilhem Y, Basseville S, Curtit F, Stéphan J-M, Cailletaud G. Investigation of the effect of grain clusters on fatigue crack initiation in polycrystals. Int J Fatigue 2010;32(11):1748-63.

[9] Przybyla CP, McDowell DL. Microstructure-sensitive extreme value probabilities for high cycle fatigue of ni-base superalloy in 100. Int J Plast 2010;26(3):372-94.

[10] Pyrz R. Correlation of microstructure variability and local stress field in twophase materials. Mater Sci Eng: A 1994;177(1-2):253-9.
[11] Fatemi A, Socie DF. A critical plane approach to multiaxial fatigue damage including out-of-phase loading. Fatigue Fract Eng Mater Struct 1988; $11: 145-65$.

[12] Meric L, Cailletaud G. Single crystal modeling for structural calculations: Part 2-Finite element implementation. J Eng Mater Technol 1991:113(1):171-82.

[13] Franciosi P, Berveiller M, Zaoui A. Latent hardening in copper and aluminium single crystals. Acta Metall 1980;28(3):273-83.

[14] Gérard C, N'Guyen F, Osipov N, Cailletaud G, Bornert M, Caldemaison D. Comparison of experimental results and finite element simulation of strain localization scheme under cyclic loading. Comput Mater Sci 2009; 46(3):755-60.

[15] Gérard C, Cailletaud G, Bacroix B. Modeling of latent hardening produced by complex loading paths in fcc alloys. Int J Plast 2013;42(0):194-212.

[16] Robert C, Saintier N, Palin-Luc T, Morel F. Micro-mechanical modelling of high cycle fatigue behaviour of metals under multiaxial loads. Mech Mater 2012:55:112-29.

[17] Crossland B. Effect of large hydrostatic pressures on the torsional fatigue strength of an alloy steel. Inst Mech Eng 1956:138-49.

[18] Matake T. An explanation on fatigue limit under combined stress. Bull JSME 1977;141:257-63.

[19] Dang Van K. Sur la résistance à la fatigue des métaux. Sciences et Techniques de l'armement, Mémorial de l'artillerie française, 3ème facicule; 1973.

[20] Dang Van K, Griveau B, Message O. On a new multiaxial fatigue limit criterion: theory and application, biaxial and multiaxial fatigue, EGF 3. London: Mechanical Engineering Publications; 1989. p. 479-96..

[21] Lukás P, Kunz L. Effect of mean stress on cyclic stress-strain response and high cycle fatigue life. Int J Fatigue 1989;11(1):55-8.

[22] Freudenthal AM, Gumbel EJ. Minimum life in fatigue. J Am Stat Assoc 1954;49:575-97.

[23] Fisher RA, Tippett LHC. Limiting forms of the frequency distribution of the largest or smallest member of a sample. Math Proc Cambridge Philos Soc $1928 ; 24: 180-90$.

[24] Jenkinson AF. The frequency distribution of the annual maxima (or minimum) values of meteorological elements. Quart J Roy Meteorol Soc 1955;81.

[25] Banvillet A, Palin-Luc T, Lasserre S. A volumetric energy based high cycle multiaxial fatigue citerion. Int J Fatigue 2003;25(8):755-69.

[26] Froustey C, Lasserre S. Multiaxial fatigue endurance of 30ncd16 steel. Int J Fatigue 1989;11(3):169-75. 